Journal of Universal Mathematics

Vol.2 No.1 PP.98-102 (2019)

ISSN-2618-5660

\title{
VARIABLE GENERALIZED CHAPLYGIN GAS AS A SCALAR FIELD DARK ENERGY MODEL
}

\author{
M. SALTI, M. KORUNUR, K. SOGUT, AND O. AYDOGDU
}

\begin{abstract}
A large number of scalar field ideas have been introduced in literature in order to discuss the speedy expansion nature of our universe. Scalar field definitions naturally come forward in particle physics. Fundamental particle physics theories can yield various scalar field descriptions, but they do not define its self-interacting potential exactly due to the complexity of corresponding equations. Here, we mainly focused on the reconstruction of the tachyon scalar field dark energy prescription by making use of the variable generalized Chaplygin gas (VGCG) dark energy model in the four-dimensional (4D) spatially flat Friedmann-Robertson-Walker (FRW) framework.
\end{abstract}

\section{INTRODUCTION}

The recent astrophysical data[1, 2, 3, 4, 5, 6] have shown that the universe has a phase transition from the decelerating era to the accelerating one and it expands faster than we expect. It is commonly accepted that an unknown mysterious component, namely the dark energy, is responsible for the recently observed speedy expansion phase of the universe. Working on the origin and the nature of dark component of our universe has been one of the great issues in modern theoretical physics. Although many ideas have been introduced in literature, the mysterious accelerated expansion phase is still completely unknown. The well-known cosmological constant (see Ref.[7] for a review), scalar fields[8, 9, 10, 11, 12], unified dark energy-matter formulations[13, 14, 15, 16], modified theories of gravity[17, 18, 19, 20, 21, 22, 23] and even assuming the existence of extra dimensions[15, 24, 25, 26, 27, 28] are possible ideas for the nature of the dark energy. Li et al.[29] and Cai et al.[30] prepared very useful briefs about the accelerated expansion behavior of the universe including a survey of some possible theoretical formulations.

In the present study, we focus on the VGCG model[31] in order to reconstruct associated potential and scalar field function defining the tachyonic scalar field dark energy model[32, 33, 34].

Date: Review January 2, 2019, accepted January 14, 2019.

2000 Mathematics Subject Classification. 95.35.+d; 95.36.+x; 98.80.k.

Key words and phrases. Cosmology, Dark Energy, Scalar Field. 


\section{Preliminaries: DARK ENERGy SCENARIO}

The VGCP unified dark matter-energy prescription is given by the following equation-of-state (EoS) [31]

$$
p=-\frac{A a^{-n}}{\rho^{\alpha}}
$$

where $p, \rho$ and $a(t)$ stand for the pressure, energy density and the cosmic scale factor, respectively. Moreover, $A, n$ and $\alpha$ are free parameters of the model. It is known that (i) $n=0$ case describes the generalized Chaplygin gas (GCG) model and (ii) the limiting condition $\alpha \rightarrow 1$ reduces the model into the variable Chaplygin gas (VCG) description.

Making use of the continuity relation $\dot{\rho}+\frac{3}{a} \frac{d a}{d t}(\rho+p)=0$, the energy density associated with the VGCG model is obtained as given below[35]

$$
\rho=\rho_{0}\left[\Delta(1+z)^{n}+(1-\Delta)(1+z)^{3(1+\alpha)}\right]^{\frac{1}{1+\alpha}}
$$

where

$$
\Delta=\frac{3(1+\alpha)}{3(1+\alpha)-n} \frac{A}{\rho_{0}^{1+\alpha}}
$$

and $\rho_{0}$ indicates the present value of the energy density. It is noteworthy to mention here that, for the speedy expansion phase, it must be[35] $n>0$ and $3(1+\alpha)>0$. Otherwise, we have $\lim _{a \rightarrow \infty} \rho \rightarrow \infty$ implying a decelerating universe. Now, in order to investigate the dynamical evolution of dark energy and dark matter and study the cosmological features of the dark energy, we rewrite the energy density of the VGCG in terms of two different contents. On this purpose, we assume $\rho=\rho_{e}+\rho_{m}$ and $p=p_{e}$ where the subscripts $e$ and $m$ indicate the dark energy and the dark matter, respectively. From this point of view, the continuity equation can be decomposed the following components:

$$
\begin{gathered}
\dot{\rho}_{e}+\frac{3}{a} \frac{d a}{d t}\left(\rho_{e}+p_{e}\right)=0, \\
\dot{\rho}_{m}+\frac{3}{a} \frac{d a}{d t} \rho_{m}=0 .
\end{gathered}
$$

Consequently, according to general acceptance of the dark matter[35], one can get

$$
\rho_{m}=\rho_{0}^{m}(1+z)^{3} \text {. }
$$

Then, it is found that [35]

$$
\begin{aligned}
\rho_{e} & =\rho-\rho_{m} \\
& =\rho_{0}\left[\Delta(1+z)^{n}+(1-\Delta)(1+z)^{3(1+\alpha)}\right]^{\frac{1}{1+\alpha}}-\rho_{0}^{m}(1+z)^{3} .
\end{aligned}
$$

As the last step, after considering the FRW spacetime dominated by the VGCG and the baryonic matter $\rho_{b}$ in order to use the Friedmann equations, the cosmic Hubble parameter $H=\frac{1}{a} \frac{d a}{d t}$ is obtained as[35]

$(2.8) H=H_{0}\left[\Omega_{0}^{e}\left\{\Delta(1+z)^{n}+(1-\Delta)(1+z)^{3(1+\alpha)}\right\}^{\frac{1}{1+\alpha}}+\Omega_{0}^{b}(1+z)^{3}\right]^{\frac{1}{2}}$.

Note that $\Omega_{0}^{e}=\frac{8 \pi G}{3 H_{0}^{2}} \rho$ and $\Omega_{0}^{b}=\frac{8 \pi G}{3 H_{0}^{2}} \rho_{b}$ are dimensionless density parameters and we have $\Omega_{0}^{e}+\Omega_{0}^{b}=1$. Next, $H_{0}$ represents the current value of the Hubble parameter. 
Furthermore, for the VGCG type dark energy, the corresponding EoS parameter $\omega_{e}=\frac{p_{e}}{\rho_{e}}$ can be written in terms of the cosmic red shift parameter as

$$
\omega_{e}=\frac{\Delta \rho_{0}^{-\alpha}(1+z)^{n}\left[\Delta(1+z)^{n}+(1-\Delta)(1+z)^{3(1+\alpha)}\right]^{-\frac{\alpha}{1+\alpha}}}{\rho_{0}\left[\Delta(1+z)^{n}+(1-\Delta)(1+z)^{3(1+\alpha)}\right]^{\frac{1}{1+\alpha}}-\rho_{0}^{m}(1+z)^{3}} .
$$

Now, we are in a position to implement a correspondence between the VGCG fluid and the tachyonic field.

\section{RECONSTRUCTION OF THE TACHYON SCALAR FIELD DESCTIPRION}

Lagrangian density describing the tachyon scalar field model is written as

$$
£_{T}=V(\phi) \sqrt{1-g^{\mu \nu} \partial_{\mu} \phi \partial_{\nu} \phi},
$$

where $V(\phi)$ denotes the self-interacting potential of tachyonic field and $g^{\mu \nu}$ is the inverse metric tensor. For the tachyon scalar field description, the corresponding energy density and pressure are defined, respectively, by[36]

$$
\begin{gathered}
\rho_{T}=\frac{V(\phi)}{\sqrt{1-\left(\frac{d \phi}{d t}\right)^{2}}}, \\
p_{T}=-V(\phi) \sqrt{1-\left(\frac{d \phi}{d t}\right)^{2}} .
\end{gathered}
$$

The tachyonic idea has been given as one of the possible description for the dynamics of dark energy and the model has very significant EoS parameter which interpolates smoothly between -1 and $0[32,37]$. Hence, the model can be used as a suitable description for the inflation at high energy as well as a source of speedy expansion behavior[33, 34].

One can write the EoS parameter for the tachyon scalar field dark energy model as given below

$$
\omega_{T}=\left(\frac{d \phi}{d t}\right)^{2}-1
$$

Note that the condition $-1<\frac{d \phi}{d t}<1$ is required in order to define a real energy density[32, 33, 34, 37]. Additionally, the corresponding constraint is $-1<\omega_{T}<0$. The tachyonic model indicates a spacetime type which has a speedy expansion, but it cannot behave like the phantom type energy[37].

After equating $\rho_{e}$ and $\rho_{T}$, one can find the following relation for the tachyonic self-interacting potential:

$$
V(\phi)=\rho_{e} \sqrt{1-\left(\frac{d \phi}{d t}\right)^{2}}
$$

and comparing equations (2.9) and (3.4), it can be written that

$$
(3.6) \frac{d \phi}{d t}=\sqrt{1+\frac{\Delta \rho_{0}^{-\alpha}(1+z)^{n}\left[\Delta(1+z)^{n}+(1-\Delta)(1+z)^{3(1+\alpha)}\right]^{-\frac{\alpha}{1+\alpha}}}{\rho_{0}\left[\Delta(1+z)^{n}+(1-\Delta)(1+z)^{3(1+\alpha)}\right]^{\frac{1}{1+\alpha}}-\rho_{0}^{m}(1+z)^{3}}} .
$$


Hence, the definition of self-interacting potential for the VGCG type tachyonic scalar field is calculated as

$$
V(\phi)=\frac{\rho_{0}\left[\Delta(1+z)^{n}+(1-\Delta)(1+z)^{3(1+\alpha)}\right]^{\frac{1}{1+\alpha}}-\rho_{0}^{m}(1+z)^{3}}{\left[\frac{\Delta \rho_{0}^{-\alpha}(1+z)^{n}\left[\Delta(1+z)^{n}+(1-\Delta)(1+z)^{3(1+\alpha)}\right]^{-\frac{\alpha}{1+\alpha}}}{\rho_{0}^{m}(1+z)^{3}-\rho_{0}\left[\Delta(1+z)^{n}+(1-\Delta)(1+z)^{3(1+\alpha)}\right]^{\frac{1}{1+\alpha}}}\right]^{-0.5}}
$$

\section{Final Remarks}

The well-known VGCG model has recently been introduced to discuss evolutionary behavior of the universe. It is commonly believed that the scalar field dark energy prescriptions may be taken into account as a useful model to understand nature of the dark universe. Thence, reconstruction of the scalar field models based on some other dark energy definitions may yield significant conclusions. This important point motivated us to construct a connection between the tachyonic scalar field dark energy model and the VGCG fluid. It is interesting to mention here that such correspondence give very important results to understand how various theoretical dark energy ideas are mutually related to each other.

\section{REFERENCES}

[1] S. Perlmutter, et al., Measurements of Omega and Lambda from 42 High-Redshift Supernovae, Astrophys. J., 517, 565 (1999).

[2] C.L. Bennet, et al., First-Year Wilkinson Microwave Anisotropy Probe (WMAP) Observations: Preliminary Maps and Basic Results, Astrophys. J. Suppl., 148, 1 (2003).

[3] M. Tegmark, et al., Cosmological parameters from SDSS and WMAP, Phys. Rev. D, 69, 103501 (2004).

[4] A.W. Allen, et al., Constraints on dark energy from Chandra observations of the largest relaxed galaxy clusters, Mon. Not. R. Astron. Soc., 353, 457 (2004).

[5] P.A.R. Ade, et al., Planck 2013 results - XII. Component separation, A\&A 571, A12 (2014).

[6] P.A.R. Ade, et al., Planck 2015 results - XIII. Cosmological parameters, A\&A 594, A13 (2016).

[7] M.F. Wondrak, The Cosmological Constant and its Problems: A Review of Gravitational Aether, e-Print: gr-qc/1705.06294

[8] V. Sahni, Dark matter and dark energy, Lect. Notes Phys., 653, 141-180 (2004).

[9] E.J. Copeland, M. Sami and S. Tsujikawa, Dynamics of dark energy, Int. J. Mod. Phys. D, 15,1753 (2006).

[10] R.R. Caldwell, A Phantom Menace? Cosmological consequences of a dark energy component with super-negative equation of state, Phys. Lett. B, 545, 23-29 (2002).

[11] B. Feng, X. L. Wang and X. M. Zhang, Dark Energy Constraints from the Cosmic Age and Supernova, Phys. Lett. B, 607, 35 (2005).

[12] T. Chiba, T. Okabe and M. Yamaguchi, Kinetically driven quintessence, Phys. Rev. D, 62, 023511 (2000).

[13] A. Yu. Kamenshchik, U. Moschella and V. Pasquier, An alternative to quintessence, Phys. Lett. B, 511, 265 (2001).

[14] J. Christensen-Dalsgard, Lecture Notes on Stellar Structure and Evolution, Aarhus Univ. Press, Aarhus, p.13 (2004).

[15] M. Salti, O. Aydogdu, H. Yanar and K. Sogut, Variable generalized Chaplygin gas in a 5D cosmology, Annals of Physics, 390, 131-142 (2018).

[16] M. Askin, M. Salti and O. Aydogdu, Cosmology via thermodynamics of Polytropic gas, Mod. Phys. Lett. A, Vol.32, No.32, 1750177 (2017).

[17] B. Boisseau, G. Esposito-Farese, D. Polarski and A. A. Starobinsky, Reconstruction of a scalar-tensor theory of gravity in an accelerating universe, Phys. Rev. Lett., 85, 2236 (2000).

[18] S. Capozziello, Curvature Quintessence, Int. J. Mod. Phys. D, 11, 483 (2002).

[19] V. Faraoni, f(R)-gravity: Successes and challenges, XVIII Congresso SIGRAV General Relativity and Gravitational Physics, Cosenza, 2225 September (2008). 
[20] S. Capozziello, R. Cianci, C. Stornaiolo and S. Vignolo, f(R) Cosmology with torsion, Phys. Scr., 78, 065010 (2008).

[21] H. Abedi and M. Salti, Multiple field modifed gravity and localized energy in teleparallel framework, Gen. Rel. Grav., 47, 93 (2015).

[22] M. Askin, H. Abedi and M. Salti, Thermodynamics in $f(T, \theta)$-gravity, Rom. J. Phys., Vol.60, Nos.1-2, 44-55 (2015).

[23] M. Salti, M. Korunur, I Acikgoz, N. Princcioglu and F. Binbay, $f(T, R)$ theory of gravity, Int. J. Mod. Phys. D, 27, 1850062 (2018).

[24] O. Klein, Quantentheorie und fünfdimensionale Relativitätstheorie, Zeits. Phys., 37, 895 (1926).

[25] T. Kaluza, On the Unification Problem in Physics, Sits. Press. Akad. Wiss. Math. Phys., K1, 895 (1921).

[26] G. Calcagni, Fractal universe and quantum gravity, Phys. Rev. Lett., 104, 251301 (2010).

[27] M. Salti, H. Yanar, O. Aydogdu and K. Sogut, Dynamics of Ghost Scalar Fields in Kaluza-

Klein Cosmology, Astrophys. Space Sci., Vol.362, No.11, 207 (2017).

[28] I. Acikgoz, F. Binbay, M. Salti and O. Aydogdu, Ghost DBI-essence in fractal geometry, Eur. Phys. J. Plus, 131, 157 (2016).

[29] M. Li, X. D. Li, S. Wang and X. Zhang, Holographic dark energy models: A comparison from the latest observational data, J. Cosmol. Astropart. Phys., 06, 036 (2009).

[30] Y.-F. Cai, S. Capozziello, M. De Laurentis and E. N. Saridakis, f(T) teleparallel gravity and cosmology, Rept. Prog. Phys., 79, 106901 (2016).

[31] X.Y. Yang, Y.B. Wu, J.B. Lu and S. Li, Evolution of Variable Generalized Chaplygin Gas, Chin. Phys. Lett., 24, 302 (2007).

[32] G.W. Gibbons, Cosmological Evolution of the Rolling Tachyon, Phys. Lett. B, 537, 1 (2002).

[33] A. Mazumdar, S. Panda and A. Perez-Lorenzana, Assisted inflation via tachyon condensation, Nucl. Phys. B, 614, 101 (2001).

[34] T. Padmanabhan, Accelerated expansion of the universe driven by tachyonic matter, Phys. Rev. D, 66, 021301 (2002).

[35] J. Lu, Cosmology with a variable generalized Chaplygin gas, Physics Letters B, 680, 404-410 (2009)

[36] M.Jamil, K. Karami and A. Sheykhi, Restoring New Agegraphic Dark Energy in RS II Braneworld, Int. J. Theor. Phys., 50, 3069 (2011).

[37] A. Pasqua, A. Khodam-Mohammadi, M. Jamil and R. Myrzakulov, Astrophys. Space Sci., 340, 199 (2012).

(Mustafa Salti)

Current address: Mersin University, Physics Department of Arts and Science Faculty, 33343, Mersin, Turkey

E-mail address: msalti@mersin.edu.tr

(Murat Korunur)

Current address: Munzur University, Tunceli Vocational College, Deparment of Electricity and Energy, 33343, Tunceli, Turkey

E-mail address: muratkorunur@yahoo.com

(Kenan Sogut)

Current address: Mersin University, Physics Department of Arts and Science Faculty, 33343, Mersin, Turkey

E-mail address: kenansogut@mersin.edu.tr

(Oktay Aydogdu)

Current address: Mersin University, Physics Department of Arts and Science Faculty, 33343, Mersin, Turkey

E-mail address: oktaydogdu@mersin.edu.trm 